\title{
ASSESSMENT OF ANTIBIOTIC PRESCRIPTION IN PATIENTS WITH NON-PNEUMONIA ACUTE RESPIRATORY TRACT INFECTION
}

\author{
RANI SAURIASARI ${ }^{1 *}$, YUSNA FADLIYYAH APRIYANTI ${ }^{1}$, DITO PRAMONO²
}

${ }^{1}$ Faculty of Pharmacy, Universitas Indonesia, Depok, 16424, Indonesia. ${ }^{2}$ Community Health Centre Sub-District Palmerah, West Jakarta, 12210, Indonesia. Email: rani@farmasi.ui.ac.id

Received: 05 April 2018, Revised and Accepted: 01 November 2018 and 30 November 2018

\section{ABSTRACT}

Objective: The Ministry of Health (MoH) Regulation No. 30, 2014, about Health Service Standards defines the need for routine monitoring of prescribing indicators for several diseases, including non-pneumonia acute respiratory tract infection (ARI).

Methods: This study compares the results of percentage analysis of the antibiotic usage in patients with non-pneumonia ARI with two methods. Medical record data from April 2016 were collected from Primary Health Care (Puskesmas) Palmerah, West Jakarta.

Results: Convenience sampling indicated that $14.28 \%$ of patients with non-pneumonia ARIs used antibiotics. Simple random sampling indicated that $25 \%$ of patients with non-pneumonia ARIs used antibiotics. Differences in sample selection methods affected the final outcome (percentage of patients who used antibiotics). The tolerance limit for antibiotic use in non-pneumonia ARIs established by MoH was $20 \%$.

Conclusion: These results indicate a need for $\mathrm{MoH}$ guidance to pay attention to the sampling techniques used in monitoring the treatment of nonpneumonia ARIs in Primary Health Care.

Keywords: Acute respiratory tract infection, Antibiotic, Non-pneumonia.

(C) 2018 The Authors. Published by Innovare Academic Sciences Pvt Ltd. This is an open access article under the CC BY license (http://creativecommons. org/licenses/by/4. 0/) DOI: http://dx.doi.org/10.22159/ijap.2018.v10s1.14

\section{INTRODUCTION}

Acute respiratory infections (ARIs) disease always ranked first among the 10 most diseases in Indonesia [1,2]. The biggest disease data on the outpatient in Indonesia, in 2009, placed upper respiratory infection in the first sequence with total cases of 488,794 , whereas hospitalized patients were ranked seventh with total cases of 36,048 [3]. The prevalence data of respiratory tract infections based on the diagnosis of health personnel and resident's complaints was 25\% in 2013 report [4].

The high prevalence of ARIs and associated effects had led to high consumption of free medicines (such as anti-influenza and cough medicine and multivitamins) and antibiotics [5]. Excessive numbers of antibiotic prescriptions are dispensed to treat patients with respiratory tract infections, especially ARIs, although most of the causes of this disease were viruses. One of the causes was the clinician's overexpectation of antibiotics, especially to prevent bacterial secondary infections, which could not be prevented [5,6]. The effects were increased bacterial resistance and an increase in unwanted side effects. It is, therefore, necessary to monitor prescriptions for non-pneumonia ARIs [6].

ARIs were the seventh most common condition among hospitalized patients [7]. The high prevalence of respiratory infection and its impact lead to increased consumption of over-the-counter drugs, such as antiinfluenza medicine, cough medicine, multivitamins, and antibiotics [8]. The Ministry of Health (MoH) Regulation No. 30 (2014), on Health Service Standards at Puskesmas regulated use of a form to monitor the number of prescriptions dispensed monthly [7]. The evaluation was intended to assess the rational use of drugs in the service system at Puskesmas. The form applies to the case of non-pneumonia ARI, nonspecific acute diarrhea, and diseases of muscle tissue (myalgia). Data were reported to the Health Service Tribe at the city/county level in each region $[1-3,9]$.
The tolerance limit for antibiotics usage in non-pneumonia ARIs was $20 \%$. However, data on monitoring and evaluation resulted in 2013 show that antibiotic user was high in non-pneumonia ARIs disease [3]. The highest use of antibiotics for non-pneumonia ARIs disease was in Nanggroe Aceh Darussalam (67.9\%), whereas Daerah Khusus Ibukota (DKI) Jakarta was 54.5\%. The highest number of prescription drugs was found in Bangka Belitung Islands (4.2), whereas DKI Jakarta was slightly above the tolerance level (2-9) [7].

Pharmacists with their pharmaceutical services could play a role in overcoming these problems by monitoring prescribing indicators. However, there was no standardization of sampling techniques for this study. Therefore, this study was conducted to compare the results of percentage analysis of the antibiotic usage in patients with nonpneumonia ARIs using the two methods; convenience sampling and simple random sampling.

\section{METHODS}

This research was an observational research with cross-sectional method, conducted in April 2016, at Pharmacy of the Puskesmas Palmerah-West Jakarta. Data were collected from the daily prescription and were transferred to the monitoring form prescribing indicator.

In thist study, we used the sample size determined by the MoH in which one prescription per day for 7 days. As comparison, we calculated sample size for proportion estimation of a known population (Fig. 1).

In total, the required sample size was 80 subjects. By adding the possibility of sample dropped out for $4 \%$, the total sample size was 84 subjects. The inclusion criteria were prescriptions containing ambroxol in April 2016 and could be read well. 


\section{Sampling technique}

The sampling technique used was convenience sampling technique (method 1) and simple random sampling technique (method 2). The research flowchart can be seen in Fig. 2.

Method 1 was a routine method at Puskesmas Palmerah. The selected sample was one prescription of non-pneumonia ARIs obtained at first, every day for 7 days. The prescribing indicator monitor form was following the filling out instruction below:

a) Patients were taken from the daily register, one case per day for each selected diagnosis. Data were collected 25 cases per selected diagnosis per month.

b) If on that day, there was no patient with the required diagnosis, the column was emptied and filled with the same diagnosis taken in the following days.

c) For each diagnosis, the patient was taken at first register on the day of recording. Diagnosis taken was single, not double, or accompanied by disease/other complaints.

d) Powder drugs and combination drugs were written with the details of the type of medicine.

e) Types of drugs included medication, injections, and external drugs.

f) Immunization was not included in the injection category.

g) The term of antibiotics included chemotherapy and anti-amoeba.

$$
\begin{aligned}
& \mathrm{n}=\frac{\mathrm{Z}^{2}{ }_{1 \cdot} \alpha_{12} \mathrm{p}(1-\mathrm{p}) \mathrm{N}}{\mathrm{d}^{2}(\mathrm{~N}-1)+\mathrm{Z}^{2}{ }_{1} \alpha_{12} \mathrm{p}(1-\mathrm{p})} \\
& \mathrm{n}=\frac{(1,96)^{2} \times 0,5(1-0,5) 482}{(0,05)^{2} \times(482-1)+(1,96)^{2} \times 0,5(1-0,5)}=80,22
\end{aligned}
$$

Fig. 1: The sample size calculation

$\mathrm{n}=$ Sample size expected, $\mathrm{p}=$ The proportion of antibiotic used in ARIs patients (set at $50 \%$ because no previous data were available), 1-p $=$ The proportion of patients that did not use the antibiotics in patients with ARIs $=1-\mathrm{P}=50 \%, \mathrm{Z1}-\alpha / 2=$ Normal standard deviation for $\alpha 5 \%=1.96, d=$ The desired level of absolute precision $=0.05, \mathrm{~N}=$ Population $=482$ h) The "compatibility with the guidelines" column was cleared. This column would be filled by the supervisor at the time of supervision visit (10 samples were taken randomly for discussion).

Method 2 was a simple random sampling technique. A total of 482 prescriptions were sorted by day, from number one to the last. It was then drawn to get a representative of 12 numbers every day for 7 days. Therefore 84 prescriptions were obtained. The draw of the script number to be taken was the prescription number, as shown in Table 1.

\section{RESULTS AND DISCUSSION}

The data studied from daily at Pharmacy. Due to the limited number of drugs available in Puskesmas, the prescription that fell into the category of samples was daily prescription containing ambroxol as the only non-pneumonial ARIs drug available at Puskesmas Palmerah. Some other supportive anti-inflammatory drugs were chlorpheniramine or loratadine, corticosteroids such as prednisone, and vitamins such as Vitamin B complex and Vitamin C. The antibiotic therapy prescribed most commonly was amoxicillin.

The sample size at Puskesmas Palmerah on April 18-24, 2016, when using convenience sampling amounted to seven prescriptions. Among all scripts, there were antibiotics $(14.28 \%)$. The total medicinal items of all prescriptions are 20 prescriptions, so the average daily drug item was 2.85 . The data could be seen in Table 2 .

The total prescription of non-pneumonial ARIs using simple random sampling showed that sample size was 84 prescriptions. Among all

Table 1: Prescription number of draw results

\begin{tabular}{ll}
\hline Date & Prescription number \\
\hline April 18, 2016 & $12,72,62,29,8,14,83,47,63,73,70,64$ \\
April 19, 2016 & $32,24,59,35,11,56,23,10,55,7,18,27$ \\
April 20,2016 & $44,25,15,51,18,4,3,42,56,5,20,12$ \\
April 21, 2016 & $57,56,3,4,64,60,50,31,44,73,7,16$ \\
April 22, 2016 & $15,39,91,22,86,5,66,18,17,21,11,69$ \\
April 23, 2016 & $50,43,42,28,51,29,1,19,36,22,4,8$ \\
April 24, 2016 & $16,36,10,6,30,37,13,47,19,24,8,1$ \\
\hline
\end{tabular}

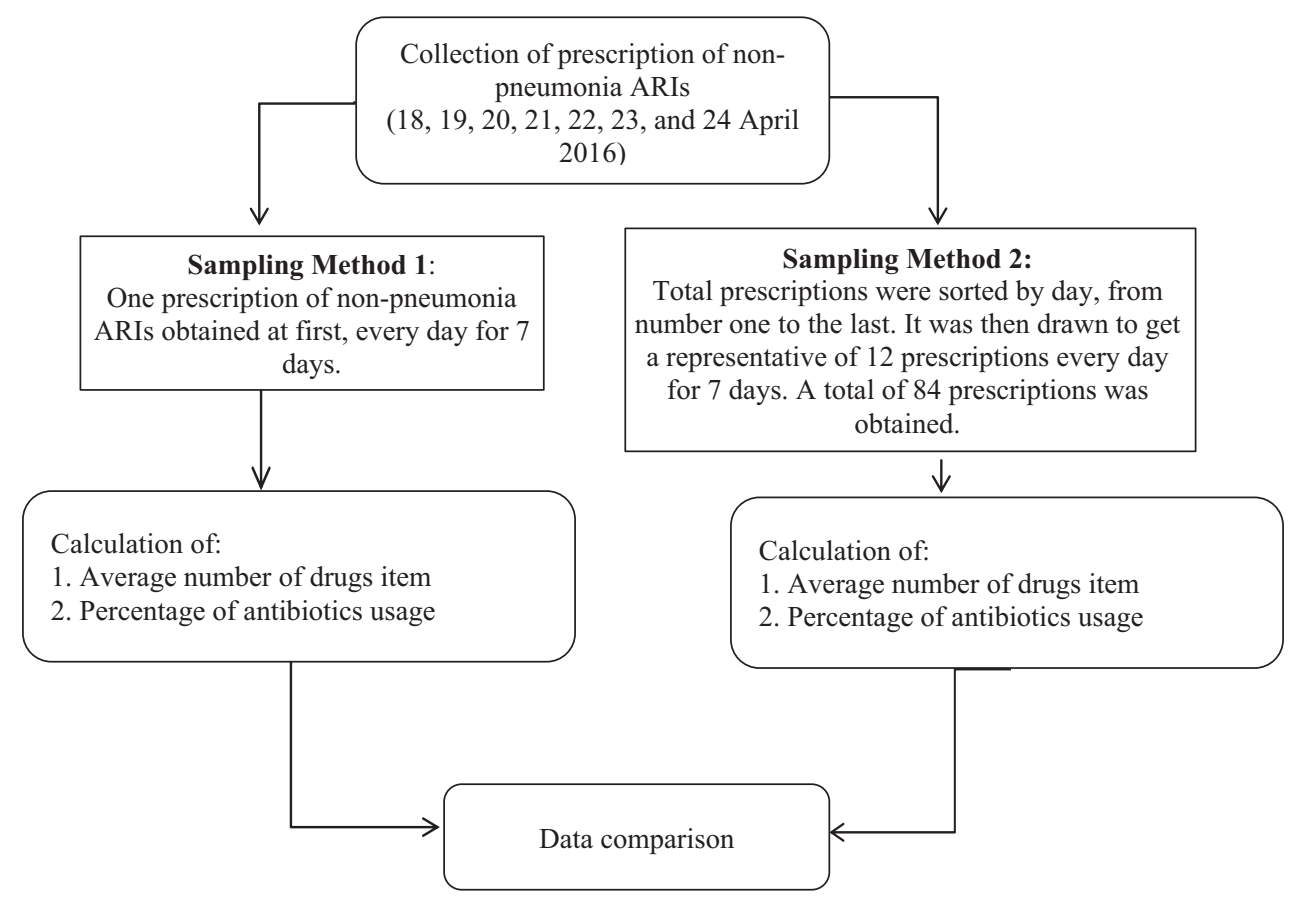

Fig. 2: Research flow chart 
prescriptions, 21 (25\%) were antibiotics, for patients diagnosed with non-pneumonial ARIs. The total drug item of all prescriptions was 247 , so the average daily drug item was 2.94 . The data could be seen in Table 3.

The World Health Organization (1985) defines the rational use of drugs as whether the patient receives a drug that suits his or her needs for an adequate period at a price affordable to him or her and the community. The irrational use of drugs is an important issue that may decrease the quality of health services by increasing microbial resistance [9].

Acute infections that attacked one or more of the respiratory tract from the nose to the alveoli include the adnexa (sinus, middle ear cavity, and pleura). Signs and symptoms of respiratory infections could include cough, difficulty in breathing, sore throat, runny nose, earache, and fever. However, airway infections therapy was not only depend on antibiotics. Some cases of acute respiratory infections were caused by viruses that did not require antibiotic therapy, but simply with supportive therapy.

According to the $\mathrm{MoH}$ protocol for management of ARIs, verification of infection is crucial before starting therapy $[1,3,9]$. This was because there were some diseases and drugs that could provide symptoms similar to infections. In addition, the use of antibiotics without evidence-based infections could lead to the increased incidence of resistance or potential drug resistance experienced by patients [10]. The symptoms of infection such as fever, leukocytosis, inflammation at the site of infection, infiltrate production from the site of infection need to be confirmed by a culture test results. Patients with non-pneumonia ARIs typically did not require antibiotic treatment.

Monitoring and evaluation of rational drug usage comprised three stages: Recording patient status, monitoring and evaluation of prescribing indicators, and collection of prescribing data [9]. The first stage was to record the patient's status. This was done to obtain preliminary patient data on patient demographic data, current patient condition, and history of patient treatment. The second stage was monitoring and evaluation of prescribing indicators. Cases included in the monitoring form of prescribing indicators were those who seek treatment at Puskesmas with a single diagnosis of non-pneumonia (colds), non-specific acute diarrhea, and muscular, and tissue system diseases (myalgia). The selection of the three diagnoses was based on the 10 most common diseases; the diagnosis could be enforced by the officer without the need for an investigation, therapeutic guidelines

Table 2: Sample data obtained with convenience sampling

\begin{tabular}{llll}
\hline No. & Date & Number of drugs item & Antibiotics usage \\
\hline 1. & April 18, 2016 & 4 & 1 \\
2. & April 19,2016 & 2 & 0 \\
3. & April 20,2016 & 4 & 0 \\
4. & April 21,2016 & 3 & 0 \\
5. & April 22, 2016 & 3 & 0 \\
6. & April 23, 2016 & 2 & 0 \\
7. & April 24, 2016 & 2 & 0 \\
& Total & 20 & 1 \\
\hline
\end{tabular}

for the three clear diagnoses, no antibiotics/injection required, and all three were considered had potency to be treated irrationally [9]. At this stage, an assessment of four prescribing indicators (median number of medicines per patient, percentage of antibiotics usage, percentage of injection used, and percentage of generic drug used) from incoming prescriptions. The third stage was collected prescribing data. Data recapitulation was conducted only after patient information had been obtained, and the patient's prescription had been assessed. The format used as reference format for data recapitulation was the form of prescription indicator monitoring conducted by filling column 1-13; column 1-9 was used for monitoring purposes; and columns 10-13 were used to assess compliance with prescribing treatment guidelines, under the supervision of the district health office.

The average number of drug items in the patients required by the directorate general of pharmaceutical and medical devices was 2.6 items as a tolerance limit. The results of the report at Puskesmas Palmerah using the first sampling method were 2.85 items, whereas when using the second sampling method was 2.94. Both data concluded that the median number of drug items administered for non-pneumonial ARIs was still above the required amount. It was very difficult to decrease the median number of medication items on non-pneumonia ARIs prescriptions, as they were often caused by allergies, which required additional drugs such as antihistamines and vitamin supplementation to speed the healing process.

The use of drugs for non-pneumonia ARI treatment was rational when convenience sampling was used but irrational when simple random sampling was used. The difference in outcomes of the percentage of antibiotic usage was seen to be very significant, due to differences in methods of sampling. Convenience sampling was less representative of the prescription per day because the sample used was very small, that is, one prescription. The retrieval technique used was also difficult to rely on because not all prescriptions had the same chance of being selected into a sample (non-probability). These techniques could save costs and time. Convenience sampling does not yet reflect the results of calculating the percentage of antibiotic used for patients with nonpneumonia ARIs.

The patient's name, age, and prescribed medications along with the number and rules of used could be obtained with valid data as they related to prescriptions brought by the patient, but for the diagnosis, the officer only wrote the patient's diagnosis regardless of the patient's status or medical records directly. The written prescription indicator form was based solely on data listed on the patient's prescription because the pharmacist often had difficulty in viewing the patient's medical status or records required for reporting. For example, the possibility of the patient had previous treatment, but 3-4 days later had not healed so that the next treatment was given antibiotics.

These issues demonstrate the need for an integrated computerized system that allows health-care practitioners to directly access patient data. If the pharmaceutical department could access patient data from a history of illness, doctor's diagnosis, laboratory results, and nutrition consultation data provided by other health workers at the Puskesmas,

Table 3: Rational drug use report data using simple random sampling

\begin{tabular}{lllll}
\hline No. & Date & Number of prescriptions & Number of drugs item & Number of antibiotics \\
\hline 1. & April 18, 2016 & 12 & 38 & 3 \\
2. & April 19, 2016 & 12 & 39 & 2 \\
3. & April 20, 2016 & 12 & 39 & 6 \\
4. & April 21, 2016 & 12 & 29 & 1 \\
5. & April 22, 2016 & 12 & 37 & 3 \\
6. & April 23, 2016 & 12 & 32 & 4 \\
7. & April 24, 2016 & 12 & 33 & 21 \\
\end{tabular}


then rational treatment would work better. Computerized data access also simplifies the workload of pharmacists and pharmacist assistants who work on the service system and reporting.

\section{CONCLUSION}

Convenience sampling does not yet reflect the results of calculating the percentage of antibiotic used for patients with non-pneumonia ARIs. Differences in the sample selection method affected the outcome of a significant percentage of antibiotic usage. Therefore, further guidance by the $\mathrm{MoH}$ related to uniformity in sampling techniques is required to monitor the treatment of non-pneumonia ARIs in Puskesmas.

\section{CONFLICTS OF INTEREST}

Authors declare no conflicts of interest in this research.

\section{REFERENCES}

1. Department of Health, Republic of Indonesia. Guidelines for Pharmaceutical Services at Puskesmas. Jakarta: Department of Health Republic of Indonesia; 2006. p. 1-36.

2. Ministry of Health, Republic of Indonesia. Rational Drug Use Module.
Jakarta: Ministry of Health, Republic of Indonesia; 2011

3. Department of Health, Republic of Indonesia. Monitoring and Evaluation of Rational Drug Use. Jakarta: Ministry of Health, Republic of Indonesia; 2010.

4. Ministry of Health, Agency for Health Research and Development. Basic Health Research. Jakarta: Ministry of Health, Republic of Indonesia; 2013.

5. Hansen MP, Hoffmann TC, McCullough AR, van Driel ML, Del Mar CB. Antibiotic resistance: What are the opportunities for primary care in alleviating the crisis? Front Public Health 2015;3:35

6. Llor C, Bjerrum L. Antimicrobial resistance: Risk associated with antibiotic overuse and initiatives to reduce the problem. Ther Adv Drug Saf 2014;5:229-41.

7. Ministry of Health, Republic of Indonesia. The Standard of Pharmaceutical Services at Community Health Center. Jakarta: Ministry of Health, Republic of Indonesia; 2014.

8. Allan GM, Arroll B. Prevention and treatment of the common cold: Making sense of the evidence. CMAJ 2014;186:190-9.

9. Ministry of Health, Republic of Indonesia. Pharmacy Management Training Material at Community Health Center. Jakarta: Ministry of Health, Republic of Indonesia; 2010.

10. Fair RJ, Tor Y. Antibiotics and bacterial resistance in the $21^{\text {st }}$ century. Perspect Medicin Chem 2014;6:25-64. 